\title{
Small GTPases and cilia
}

\author{
Yujie $\mathrm{Li}^{1}$, Jinghua $\mathrm{Hu}^{1,2,3 凶}$ \\ ${ }^{1}$ Department of Nephrology and Hypertension, Mayo Clinic, Rochester, Minnesota, USA \\ 2 Translational PKD Center, Mayo Clinic, Rochester, Minnesota, USA \\ ${ }^{3}$ Department of Biochemistry and Molecular Biology, Mayo Clinic, Rochester, Minnesota, USA \\ $\triangle$ Correspondence: Hu.jinghua@mayo.edu \\ Received January 3, 2011 Accepted January 6, 2011
}

\begin{abstract}
Small GTPases are key molecular switches that bind and hydrolyze GTP in diverse membrane- and cytoskeletonrelated cellular processes. Recently, mounting evidences have highlighted the role of various small GTPases, including the members in Arf/Arl, Rab, and Ran subfamilies, in cilia formation and function. Once overlooked as an evolutionary vestige, the primary cilium has attracted more and more attention in last decade because of its role in sensing various extracellular signals and the association between cilia dysfunction and a wide spectrum of human diseases, now called ciliopathies. Here we review recent advances about the function of small GTPases in the context of cilia, and the correlation between the functional impairment of small GTPases and ciliopathies. Understanding of these cellular processes is of fundamental importance for broadening our view of cilia development and function in normal and pathological states and for providing valuable insights into the role of various small GTPases in disease processes, and their potential as therapeutic targets.
\end{abstract}

\section{KEYWORDS Small GTPase, cilia, ciliopathy}

\section{INTRODUCTION}

Cilia act as motile or sensory devices on the surfaces of most eukaryotic cells, and cilia dysfunction results in a variety of severe human pathologies, now collectively termed ciliopathies (Badano et al., 2006; Singla and Reiter, 2006; Fliegauf et al., 2007; Marshall, 2008). Phylogenetically conserved intraflagellar transport (IFT) machinery, which is composed of IFT$A$ and IFT-B subcomplexes, mediates the bidirectional movement of IFT cargos that are required for the biogenesis, maintenance, and signaling of cilia (Rosenbaum and Witman, 2002; Scholey and Anderson, 2006; Pedersen and
Rosenbaum, 2008; Scholey, 2008). However, an understanding of how cilia form and execute their sensory function remains poorly defined.

In the last few years, several lines of emerging evidences suggested that small GTPases could play critical roles in many aspects of cilia formation and signaling. The small GTPase superfamily has numerous members. In this review, we focus on several small GTPases in Arf/Arl, Rab, and Ran subfamilies. We will briefly introduce the properties of small GTPases and the current understanding about cilia and ciliopathies. We will discuss the recent advances that revealed the importance of various small GTPases in the context of cilia, with an emphasis on the underlying molecular mechanism and the general implication in cilia biogenesis and sensory function. Lastly, we will introduce the advantages of applying the genetic model nematode Caenorhabditis elegans in cilia-related research. Our goal in this review is to highlight the critical features of small GTPases in cilia-related cellular events in both normal and pathological states.

\section{SMALL GTPases}

The small GTPase superfamily is divided into five major subfamilies: Ras, Rho, Rab, Arf/Arl, and Ran, on the basis of sequence and function similarities (Wennerberg et al., 2005). Small GTPases are key regulators of many cellular processes, such as vesicular trafficking, differentiation, cell division, proliferation, migration, cytoskeleton organization, gene transcription, and nuclear assembly (Wennerberg et al., 2005; Lundquist, 2006). Like heterotrimeric $G$ proteins, small GTPases are monomeric $G$ proteins and act as molecular switches, where the switching process relies on GTP hydrolysis. Small GTPases cycle between the active GTPbound and the inactive GDP-bound states (Boguski and McCormick, 1993; Donovan et al., 2002). The GTP/GDP cycling process is facilitated by guanine nucleotide exchange factors (GEFs) that both stimulate GDP loss and GTP 
binding, as well as by GTPase activating proteins (GAPs) that mediate GTP hydrolysis (Cherfils and Chardin, 1999; Donovan et al., 2002; Bernards and Settleman, 2004).

The founding members of small GTPase superfamily are the Ras proteins that were first characterized as human oncogene products (Lowy and Willumsen, 1993). As master regulators of diverse signaling pathways, Ras proteins control cell proliferation, differentiation and survival (Reuther and Der, 2000; Wennerberg et al., 2005). Small GTPases in Rho subfamily mainly serve as key regulators in actin and microtubule cytoskeleton organization, cell cycle progression, cell division, adhesion and gene expression (Jaffe and Hall, 2005). Rab proteins comprise the largest subfamily of small GTPases with more than 60 members in mammals (PereiraLeal and Seabra, 2001; Zerial and McBride, 2001; Stenmark, 2009). They are believed to control the membrane identity, vesicle budding, uncoating, tethering and fusion through the recruitment of the sorting, tethering and docking factors (Stenmark, 2009). Like the Rab proteins, the ADP-ribosylation factor (Arf) and some Arf-like (Arl) proteins are also involved in vesicular transport (D'Souza-Schorey and Chavrier, 2006; Myers and Casanova, 2008). Ran is the most abundant small GTPase in the cell, which is known for its nucleocytoplasmic transport function of both proteins and RNAs (Weis, 2003).

\section{CILIA AND CILIOPATHIES}

Cilia, specialized organelles with a microtubule core (the axoneme), are present on the surfaces of most polarized cells in the human body. There are two main types of cilia: motile and immotile (also known as primary cilia). In general, motile cilia contain 9 pairs of outer microtubule doublet surrounding a central pair $(9+2$ configure). The dynein arms and radial spoke are connected to outer doublets and responsible for generating force and regulating the ciliary beating direction. Primary cilia lack the central pair and present a $9+0$ arrangement. While the physiologic roles of motile cilia in cell locomotion, sexual reproduction and fluid flow generation have long been recognized, the importance of primary cilia was overlooked until last decade when the correlation between the primary cilia dysfunction and various human genetic diseases was revealed.

Primary cilia serve as sensory organelles and assemble via the IFT, two features that are phylogenetically conserved in all ciliated organisms. IFT is a microtubule-based bi-directional motility that is required for the development of all cilia/flagella (Rosenbaum and Witman, 2002; Pedersen and Rosenbaum, 2008). Pioneering insights into the mechanism of IFT emerged from the observation of microscopic particles moving up and down the length of flagella of the green algae Chlamydomonas (Kozminski et al., 1998). The cellular machinery that drives IFT was determined primarily by cellular and biochemical approaches (see references within (Rosen- baum and Witman, 2002)). The IFT machinery in Chlamydomonas includes heterotrimeric kinesin-II, which consists of two motor subunits and one non-motor accessory subunit, and retrograde cytoplasmic dynein motors that move IFT particles and cargos to and from the distal tips of cilia. The IFT particle is composed of two subcomplexes ( $A$ and $B$ ) that contain 16-18 polypeptides. It's well accepted that the IFT complex is assembled at the cilia base to load the cargos, including membrane receptors, structural proteins, and signaling molecules, then transported to the ciliary tip and unloaded the cargos, which are used to build cilia and maintain the sensory function (Rosenbaum and Witman, 2002; Scholey and Anderson, 2006; Pedersen and Rosenbaum, 2008; Scholey, 2008). On the other hand, retrograde movement of the IFT complex not only functions to recycle the IFT machinery to ciliary base, but also is indispensable for the cilia signaling transduction, such as phototaxis in green algae (Lechtreck et al., 2009) and sonic hedgehog $(\mathrm{Hh})$ signaling in mammalian cells (Huangfu and Anderson, 2005; May et al., 2005; Tran et al., 2008).

Consistent with their universal cellular distributions and their roles in a number of pivotal signaling pathways, such as Hh signaling, platelet-derived growth factor receptor (PDGFR) signaling, Wnt signaling, and Planar Cell Polarity $(\mathrm{PCP})$, perturbations in the cilia formation and function give rise to a broad range of genetic disorders, collectively termed ciliopathies (Badano et al., 2006; Gerdes et al., 2009; Goetz and Anderson, 2010). This classification includes polycystic kidney disease (PKD), Bardet-Biedl syndrome (BBS), Joubert syndrome, nephronophthisis (NPHP), Meckel-Gruber syndrome (MKS) and so on (Table 1). The functions of all identified ciliopathy candidates have been suggested to be cilia and/or basal body related; nonetheless, the precise roles remain enigmatic (Eley et al., 2005; Badano et al., 2006; Fliegauf et al., 2007).

\section{SMALL GTPases AND CILIOPATHIES}

In the past decade, a large body of evidences revealed that small GTPases are involved in cilia biogenesis and function, as well as ciliopathies.

\section{Arf/Arl}

The cellular functions of most Arf/Arl small GTPases are poorly understood. The first direct evidence connected an inherited human ciliopathy with a member of Arf/Arl small GTPases came from Arl6 (also termed BBS3) (Fan et al., 2004). BBS is a genetically heterogeneous human disorder characterized by obesity, polydactyly, mental retardation, retinal degeneration, and renal cyst (Zaghloul and Katsanis, 2009). So far, 14 BBS loci have been found in patients. To map the causal gene in BBS3 patients, Fan et al. first reasoned that the ortholog of $B B S 3$ should exist in other 
Table 1 Clinical feature in the ciliopathies

\begin{tabular}{|c|c|c|c|c|c|c|c|c|c|}
\hline & PKD & BBS & MKS & JS & NPHP & OFD & $\mathrm{SL}$ & ALMS & MKKS \\
\hline Cystic kidney & + & + & + & + & + & + & + & + & \\
\hline Heart disease & & + & & & & & & + & + \\
\hline Hepatic dysfunction & + & + & + & + & + & + & + & + & \\
\hline Retinal degenaration & & + & + & + & & & + & + & \\
\hline Mental retardation & & + & + & + & & + & & & \\
\hline Polydactyly & & + & + & + & & + & & & + \\
\hline Diabetes & & + & & & & & & + & \\
\hline Obesity & & + & & & & & & + & \\
\hline Left-right asymmetry & & + & + & + & + & & + & & \\
\hline
\end{tabular}

PKD, polycystic kidney disease; BBS, Bardet-Biedl syndrome; MKS, Meckel-Gruber syndrome; JS, Joubert syndrome/Cerebello-oculo-renal syndrome; NPHP, nephronophthisis; OFD, orofaciodigital syndrome; SLSN, Senior-Løken syndrome; ALMS, Alstrom syndrome; MKKS, McKusick-Kaufman syndrome.

ciliated organisms. In C. elegans, it's already known that daf19 encodes the sole member of the regulatory factor $X$ (RFX) transcription factor family and acts as the master regulator of ciliogenesis (Swoboda et al., 2000). DAF-19 regulates ciliogenesis by binding to an X-box motif in the promoters of target genes. By analyzing the $C$. elegans genes with $X$-box in their promoters and then correlating to the human gene homologs in the BBS3 critical interval, Fan et al., were able to identify ARL6 as the causal locus for BBS3 (Fan et al., 2004). A similar comparative genomics approach using $C$. elegans genome as reference also characterized ARL6 as the gene mutated in BBS3 patients (Chiang et al., 2004). With the determination of the crystal structure of GTP-bound Arl6 (Wiens et al., 2010), it's found that the altered resides found in BBS3 patients cluster in or around Arl6 GTP binding motif, suggesting the importance of small GTPase activity in ciliopathy etiology. Furthermore, overexpression of GDP- or GTP-locked forms, leads to anomalies in cilia assembly and Wnt signaling in vivo (Wiens et al., 2010). Seven other BBS proteins (BBS1, BBS2, BBS4, BBS5, BBS7, BBS8, and BBS9) and a BBIP10 protein form the core complex called the BBSome (Nachury et al., 2007; Loktev et al., 2008). The BBSome is proposed in promoting cilia membrane biogenesis (Nachury et al., 2007), and the ciliary targeting of IFT cargo proteins (Berbari et al., 2008; Jin et al., 2010). Nachury's group found that Arl6 ${ }^{\text {GTP }}$ and the BBSome localize to mammalian primary cilia in an interdependent manner, and the ciliary targeting of the membrane receptor somatostatin receptor 3 (SSTR3CTS) is directly mediated by the BBSome (Jin et al., 2010). This finding supports the hypothesis that the abnormal Arl6 small GTPase activity leads to the compromised ciliary entry of critical cargo proteins essential for cilia assembly and signaling and, thus, causes the variety of BBS symptoms.

The second Arl small GTPase identified as a ciliopathy candidate is $A r l 13 b$, one of causal loci for ciliopathy Joubert Syndrome disorder. Joubert syndrome is the most common inherited cerebellar malformation syndrome and characterized by congenital cerebellar ataxia, hypotonia, oculomotor apraxia, and mental retardation, cystic kidney, polydactyly. Unlike the common $\sim 25 \mathrm{kDa}$ small GTPase, Arl13b is an atypical small GTPase with an elongated $C$ terminus which contains a coiled-coil domain and a proline-rich domain. Similar to BBS3, mutations found in JS patients interfere with the GTP binding activity of Arl13b. Arl13b mutant mice (hennin) showed coupled defects in cilia structure and $\mathrm{Hh}$ signaling, and mimicked the mutant phenotypes in human patients (Caspary et al., 2007). Due to the lethality of hennin mice, $C$. elegans is used as an alternative model to gain mechanistic insights into the in vivo function of Arl13b protein. Studies from the Blacque's laboratory and our laboratory revealed a role for $A R L-13$, the $C$. elegans ortholog of human Arl13b, at ciliary membranes, where it regulates ciliary transmembrane protein localizations and anterograde IFT assembly stability (Cevik et al., 2010; Li et al., 2010). Moreover, our laboratory's work showed that ARL-3, the C. elegans homolog of human Arl3, acts antagonistically with ARL-13 to regulate IFT integrity and ciliogenesis ( $\mathrm{Li}$ et al., 2010). Specifically, the absence of ARL-13 causes destabilized IFT complex and IFT-A and IFT-B tend to dissociate in anterograde IFT transport; whereas $\mathrm{ARL}-3$ depletion can partially rescue ciliogenesis defects in arl-13 mutants by stabilizing the association between IFT-A and IFT-B.

Our work revealed that ARL-3 acts through an HDAC6dependent pathway ( $\mathrm{Li}$ et al., 2010). HDAC6 is a unique member of the histone deacetylase family because it deacetylates not only histone (Grozinger et al., 1999), but also a-tubulin (Hubbert et al., 2002), Hsp90 (Kovacs et al., 2005), and coractin (Zhang et al., 2007). Inhibition of HDAC6 enzymatic activity causes increased kinesin-1-cargo complex transportation along the microtubule in neuronal cells (Reed et al., 2006). Interestingly, activation of HDAC6 was found to promote cilia disassembly and loss of HDAC6 activity selectively stabilizes cilia in human retinal epithelial cells 
(Pugacheva et al., 2007). Currently, HDAC6 is thought to regulate microtubule-dependent cellular processes by deacetylating a-tubulin. However, this model is not true at least in C. elegans ciliogenesis pathway because MEC-12, the only a-tubulin capable of being acetylated, does not express in ciliated cells in C. elegans (Fukushige et al., 1999). Another HDAC6 substrate Hsp90 was shown to associate with $\beta$ tubulin and may be involved in stabilizing tubulin polymerization in cilia (Takaki et al., 2007). However, a null allele of daf21 , the $C$. elegans homolog of $H s p 90$, possesses normal cilia in our analysis (data not shown). These data ruled out the possibilities of $\alpha$-tubulin and Hsp90 as the candidates in ARL3-HDAC-6 pathway in the process of cilia formation in $C$. elegans. Based on our model, we propose that HDAC- 6 probably influences the acetylation level of an unknown adaptor protein in IFT complex. Intriguingly, HDAC6 was found physically associated with a subunit of the BBSome, BBIP10 (Loktev et al., 2008), suggesting a potential functional crosstalk between Arl3 and Arl6/BBS3 pathways in cilia.

The cilia-related function for Arl3 and Arl6 may be highly conserved across species. A comparative genomics study indicated that Arl3 and Arl6 are the only two small GTPases presented in the genome of all ciliated organisms (AvidorReiss et al., 2004). An Arl3 homolog product was found to target to the Leishmania flagella and proved to be essential for flagellum biogenesis (Cuvillier et al., 2000). Arl $3^{-/-}$mice failed to thrive after birth, and all died by 3 weeks of age (Schrick et al., 2006). Although Arl3 is not identified as a ciliopathy gene, $\mathrm{Ar} / 3^{-/-}$mice do exhibit classic phenotypes of a ciliopathy such as retinal degeneration, cyst formation in kidney, liver and pancreas as well as epithelial cell proliferation (Schrick et al., 2006). It is also reported that Arl3 localized predominantly to connecting cilia in mammalian rod and cone photoreceptors (Grayson et al., 2002). Arl3 has been shown to interact with the $X$-linked retinitis pigmentosa protein (RP2), which is demonstrated to act as the GAP for Arl3 (Grayson et al., 2002). RP2 localizes to the basal body and the associated centriole at the base of the photoreceptor cilium. Mutations in RP2 lead to a clinically and genetically diverse group of retinal dystrophies characterized by progressive photoreceptor degeneration. In photoreceptor cells, RP2 is proposed to regulate Arl3 small GTPase activity and facilitate the vesicle transport from Golgi to the base of the photoreceptor connecting cilia (Evans et al., 2010).

Taken together, Arl3, Arl6, and Arl13b are three conserved small GTPases implicated in human ciliopathies or vertebrate ciliopathy models. To date, the cellular effectors of Arl3, Arl6 and Arl13b are poorly understood. However, experiments on various Arl mutants with altered GTP binding capacity indicate that normal ciliogenesis requires the GTPase activities of the three ciliary Arls. Identification of the putative GEF, GAP and other Arl binding effectors will be critical for fully dissecting their roles in cilia biogenesis and signalings. It will be also interesting to investigate how the three Arl proteins coordinate their functions in cilia. The abilities of small GTPases to bind different partners and effectors and cycle between an inactive GDP-bound state and an active GTP-bound state make them promising candidates for molecular switches that regulate decisive steps in cilia biogenesis and/or signal transduction. The severe phenotypes related to cilia dysfunction in the aforementioned $\mathrm{Arl}$ ciliopathy models support this assertion. Thus, determining the functions of the Arls and their bona fide effectors will advance our understanding of cilia formation and function, as well as of ciliopathy pathogenesis.

Other than Arl proteins, Arf4 is the only reported Arf protein that shows cilia-related function (Mazelova et al., 2009a). Arf4 binds specifically to rhodopsin VxPx motif, a functional cilia targeting motif that existed in several cilia membrane proteins (Deretic et al., 2005), including polycystin-2 (PC2) (Geng et al., 2006), a protein affected in autosomal dominant polycystic kidney disease (ADPKD), and the cyclic nucleotide-gated (CNG) channel CNGB1b subunit in olfactory neurons (Jenkins et al., 2006). Upon the binding of Arf4 with the rhodopsin VxPx motif, a complex including Arf4, Arf4 GAP protein ASAP1, Arf4 effector FIP2, and another small GTPase Rab11, forms to regulate the transport of rhodopsin from the Trans-Golgi-Network (TGN) to photoreceptor cilia (Mazelova et al., 2009a). If Arf4 plays a pivotal role in sorting rhodopsin out of the TGN and into carrier vesicles that are targeted to the cilium, it would be interesting to test whether Arf4 also mediates the ciliary targeting of other ciliary sensory receptors such as PC-2, CNG, and Smoothened (Smo).

\section{Rabs}

Rab proteins form the largest family of small GTPases superfamily. Given the general role of Rab proteins in mediating membrane trafficking and imparting identity to the subdomains of the cell surface, it is likely that specific Rab protein functions in membrane trafficking to primary cilia. The first study that linked the function of Rab small GTPases to cilia came from analyses of Rab8 in photoreceptor cells. It has been found that, in photoreceptor rod cells, more than $10 \%$ of the total cellular Rab8 protein localizes on the TGN and may participate in rhodopsin cilium entry (Deretic et al., 1995). This finding was later confirmed by the fact that mutant Rab8 causes defects in the trafficking of rhodopsin-carrying postGolgi vesicles to the outer segment of retinal photoreceptor, and this process is mediated by the exocyst and SNARE proteins (Moritz et al., 2001). Other than rhodopsin, Rab8 is also implicated in cilium entry of other cilia membrane proteins. For example, Rab8 physically associates with and mediates the ciliary targeting of the cilia membrane protein fibrocystin, the gene product of human ciliopathy ARPKD (Follit et al., 2010); additionally, the GTPase activity of Rab8 is required for the cilium entry of $\mathrm{Hh}$ signaling receptor Smo (Boehlke et al., 2010). Furthermore, a mechanistical insight 
about Rab8's cilium-related role was obtained by the studies from Nachury and his colleagues. Nachury et al. proposed that the BBSome recruits Rab8 via its GEF, Rabin8, to direct post-Golgi vesicles fusion at the cilia base (Nachury et al., 2007). Rabin 8 acts to promote the GTP loading on Rab8 and Rab8 ${ }^{\text {GTP }}$ may drive the docking and fusion of exocytic vesicles to the base of the ciliary membrane. Notably, Omori et al. demonstrated that Rabaptin5, a well studied Rab5 effector involved in endocytosis (Stenmark et al., 1995), functions together with Rab8 and IFT component Elipas/DYF11 as a bridging system between the IFT complex and the IFT cargo that assemble at the cilia membrane (Omori et al., 2008). Additionally, CEP290, the gene product of a ciliopathy Joubert Syndrome causal locus, localizes around the cilia base and interacts with Rab8 (Tsang et al., 2008). Depletion of CEP290 interferes with localization of Rab8 to cilia and compromises the normal ciliogenesis (Kim et al., 2008).

Upstream of Rab8 signaling cascade is its GEF, Rabin8. Intriguingly, the GTP-bound Rab11 is found to physically interact with Rabin8 and regulate ciliogenesis, and Rab11 ${ }^{\text {GTP }}$ can stimulate the GEF activity of Rabin8 toward Rab8 (Knödler et al., 2010). Rab11 and the BBSome bind to the same region of Rabin8, and activated Rab11 seems to promote the association of the BBSome with Rabin8 (Knödler et al., 2010). As mentioned above, Rab11 and Arf4 form a ternary complex with Arf effector FIP3, and the Arf GAP ASAP1, and are all required for the packaging of rhodopsin into carrier vesicles from isolated Golgi membranes (Mazelova et al., 2009a). Taken together, these observations propose a working model in which Rab11 and Arf4 acts at the TGN to recruit and activate Rab8, and the activated Rab8 is anchored to the cilia base by CEP290 and mediate the docking and fusion of TGN vesicles to cilia membrane (Fig. 1).

Recently, Rab10 emerges as a new Rab involved in ciliogenesis. Rab10 is another mammalian homolog of yeast Sec4p that is closely related to Rab8 and has likewise been associated with cilia-related membrane transport pathways. Studies showed that Rab10 physically interacts with the exocyst complex protein Sec8 and colocalizes with the exocyst at the cilia base (Babbey et al., 2010). The exocyst, an evolutionarily conserved octameric protein complex ( $\mathrm{He}$ and Guo, 2009), has increasingly been implicated to play a role in cilia. Proteomic analysis indicates most exocyst proteins can be found in the mouse photoreceptor cilium (Liu et al., 2007). Sec10 has been shown to be necessary for ciliogenesis and dysfunction of Sec10 leads to cyst formation in kidney epithelial cells (Zuo et al., 2009). Two other exocyst components, Sec6 and Sec8, localize at the cilia base in MDCK cells (Rogers et al., 2004; Babbey et al., 2010). The exocyst complex has been suggested as an effector for Rab8 in cilia targeting of rhodopsin (Mazelova et al., 2009b). However, knockdown of Rab10 didn't produce consistent defects in ciliogenesis (Babbey et al., 2010). Considering the sequence homology between Rab8 and Rab10 and that they both use the exocyst as the effector, it could be possible that Rab10 and Rab8 are redundant players regulating the same cellular process at the cilia base. Interestingly, a number of other small GTP binding proteins have been demonstrated to directly interact with the exocyst. For example, Sec15 is the downstream effector of Sec4p in yeast and of Rab11 in higher eukaryotes that regulate the corresponding membrane trafficking stages (Guo et al., 1999; Zhang et al., 2004; Wu et al., 2005; Oztan et al., 2007); ARF6 binds Sec10 directly and uses it as downstream effector (Prigent et al., 2003; Fielding et al., 2005). These evidences suggest that the exocyst seems to function as a hub for receiving regulatory information from various small GTPase-meditated pathways. Thus, it would be interesting to examine whether and how the exocyst-associating small GTPases coordinate their functions in ciliogenesis.

By overexpressing 39 predicted human Rab GAPs, Yoshimura et al. found that XM_037557, TBC1D7, and EVI5like (the GAPs of Rab8, 17, and 23, respectively) are involved in primary cilium formation (Yoshimura et al., 2007). Little is known about the cellular function of Rab17. While Rab23 has been discovered as a negative regulator of $\mathrm{Hh}$ signaling (Eggenschwiler et al., 2006), and mutations in Rab23 lead to Carpenter's syndrome, which has overlapped phenotype with ciliopathies (e.g. polydactyly and obesity) (Jenkins et al., 2007). A recent study showed that Rab23 is present in primary cilia and depletion of Rab23 or expression of dominant-negative Rab23 increases the fraction of $\mathrm{Hh}$ receptor Smo inside cilia, suggesting a role of Rab23 in Smo turnover in the cilium (Boehlke et al., 2010).

Two Rab like proteins Rab-like 5 (IFTA-2) and Rab-like 4 (IFT27) are involved in cilia/flagellar function in C. elegans and Chlamydomonas reinhardtii, respectively (Schafer et al., 2006; Qin et al., 2007). Rabl4 and Rabl5 are unique Rab-like small GTPases because they lack the C-terminal prenylation motifs, which are essential membrane targeting signals for all Rabs. Therefore, Rabl4 and Rabl5 must function differently when compared to the canonical Rabs. Accordingly, unlike other Rabs that regulate membrane trafficking in ciliogenesis, both Rabl4/IFT27 and Rabl5/IFTA-2 appear to be components in IFT machinery and exhibit both anterograde and retrograde IFT motility in cilia. Rabl4/IFT27 is found to be instrumental in maintaining the stability of IFT complexes and contributes to flagella assembly (Qin et al., 2007). However, disruption of Rabl5/IFTA-2 shows no defect in cilium formation, but rather affects specific cilium signaling activities (Schafer et al., 2006). This observation raises an intriguing hypothesis that Rabl5/IFTA-2 may act as an adaptor protein for anchoring the signaling molecules whose signaling transduction is dependent on the IFT transport.

Although no Rab is identified as ciliopathy gene yet, they are indeed involved in different steps in ciliogenesis from membrane trafficking, vesicle fusion, to IFT machinery 


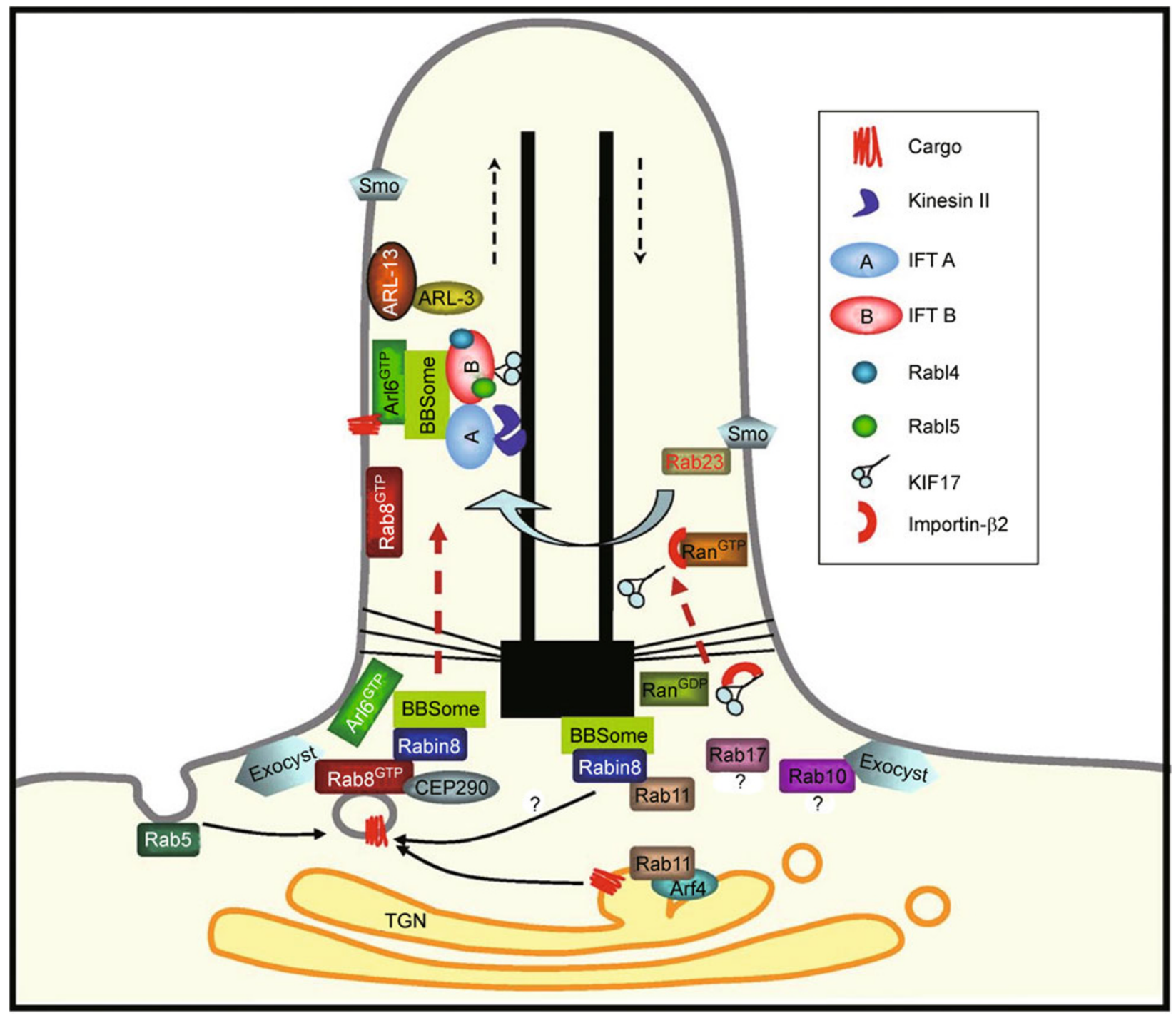

Figure 1. A model for the roles of different small GTPase proteins in cilia. Various GTPases are involved in ciliary protein transport. After activation by the Rabin8, the GTP-bound Rab8 small GTPase is proposed to facilitate docking and fusion of vesicles bearing transmembrane proteins near the ciliary membrane. The BBSome probably first docks onto the intraflagellar transport (IFT) machinery present at the transitional fibers and then moves into the cilia along with Rab8 ${ }^{\text {GTP }}$. Rab 11 stimulates the GEF activity of Rabin8 toward Rab8 and act upstream of Rab8. Smo enters the ciliary compartment via Rab8. Upon exit from the cilium, Smo is recycled back by Rab23. ARL-13 and ARL-3 are proposed to coordinate the IFT complex integrity. RanGTP/GDP gradient is critical for ciliary protein import.

regulation. And functional correlations between Rabs and ciliopathy gene products are also demonstrated in several studies. The future work on the molecular mechanism underlying Rabs in cilia will definitely enhance our understanding of ciliogenesis as well as of ciliopathy pathogenesis.

\section{Ran}

The small GTPase Ran plays roles in nuclear transport, mitotic spindle assembly and nuclear envelope assembly (Zheng, 2004; Stewart, 2007; Clarke and Zhang, 2008). It has become clear that many of these processes rely on Ran GTP binding proteins of the importin- $\beta$ superfamily (Stewart, 2007). Two independent studies showed that Ran and importin proteins are present in ciliary proteomes (Gherman et al., 2006; Liu et al., 2007). Consistent with this, Dishinger et al. found that Ran and importin- $\beta 2$ regulate the ciliary entry of kinesin2 motor KIF17 in a similar manner of nuclear transport (Dishinger et al., 2010). GTP-bound Ran is present on primary cilia. The ciliary localization signal (CLS) identified in the KIF17 is similar to classic nuclear localization signals (NLSs) and is necessary and sufficient for cilium targeting. 
Like nuclear transport, high levels of Ran ${ }^{\text {GTP }}$ are present in the cilium and the ciliary-cytoplasmic gradient of Ran regulates the ciliary entry of kinesin motor KIF17 (Dishinger et al., 2010). This is the first evidence demonstrating that $\operatorname{Ran}^{\mathrm{GTP}} / \mathrm{Ran}^{\mathrm{GDP}}$ gradient across the ciliary/cytoplasmic barrier regulates ciliary import. Whether and how this pathway regulates the entry of other motors and their cargoes at the cilia base need to be further characterized.

\section{PERSPECTIVES}

Cilia are specialized organelles whose biogenesis and maintenance require the precisely spatiotemporal integration of cytoskeleton dynamics and polarized membrane trafficking. It's not at all surprising to see that more and more small GTPases, a group of key switches involved in membrane and cytoskeleton-related cellular processes are found to play roles in ciliogenesis (Fig. 1 and Table 2). However, most of our understandings about the correlation between small GTPases and cilia are still in their infant stage. As we move forward, the molecular mechanisms underlying the ciliary roles of small GTPases must be further pursued to define the framework in this new research field. To achieve this longterm goal, we need first determine the full parts list of small GTPases involved in cilia biogenesis and function. Although Yoshimura and his colleagues have examined all Rab small GTPases by checking if the overexpression of a particular Rab GAP can compromise the ciliogenesis (Yoshimura et al., 2007), this approach is actually indirect and non-conclusive due to several pitfalls: for example, unidentified Rab GAPs may exist; GAP overexpression level may be not efficient enough to block Rab ${ }^{\text {GTP }}$ activity; two or more Rabs may play redundant roles in one step in ciliogenesis; some Rabs may play a negative role in ciliogenesis. Direct analysis on individual small GTPase, including Rabs, Arfs/Arls, and beyond, by high-throughput or case-by-case approach, need to be done in the future.

Studies of small GTPase families have demonstrated that numerous proteins bind to small GTPases to control their localization, activity, and downstream signaling pathways. The interactors include enzymes involved in posttranslational modifications, guanine nucleotide exchange factors (GEFs),

Table 2 Small GTPases and cilia

\begin{tabular}{|c|c|c|c|c|}
\hline $\begin{array}{l}\text { Small } \\
\text { GTPases }\end{array}$ & Organism & Localization & Function & References \\
\hline$\overline{A r l 13 b}$ & $\begin{array}{l}\text { C. elegans } \\
\text { Zebrafish } \\
\text { Human, mouse }\end{array}$ & $\begin{array}{l}\text { middle segment of cilia } \\
\text { Cilia } \\
\text { Cilia }\end{array}$ & Cilia formation & $\begin{array}{l}\text { Cevik et al., 2010; Li et al., 2010; } \\
\text { Duldulao et al., 2009; } \\
\text { Cevik et al., 2010; Hori et al., } 2008\end{array}$ \\
\hline Arl6 & $\begin{array}{l}\text { C. elegans } \\
\text { Human }\end{array}$ & $\begin{array}{l}\text { Ciliated cell and cilia } \\
\text { Cilia (ATP-bound form) }\end{array}$ & Cilia formation & $\begin{array}{l}\text { Fan et al., 2004; } \\
\text { Jin et al., } 2010\end{array}$ \\
\hline Arl3 & $\begin{array}{l}\text { Leishmania } \\
\text { C. elegans } \\
\text { Mouse }\end{array}$ & $\begin{array}{l}\text { Flagella } \\
\text { Ciliated cell and cilia } \\
\text { Cilia }\end{array}$ & $\begin{array}{l}\text { Flagella and cilia } \\
\text { formation }\end{array}$ & $\begin{array}{l}\text { Cuvillier et al., } 2000 \text {; } \\
\text { Li et al., 2010; } \\
\text { Zhou et al., } 2006\end{array}$ \\
\hline Arf4 & $X$. laevis. & Golgi & $\begin{array}{l}\text { Ciliary protein } \\
\text { targeting }\end{array}$ & Deretic et al., 2005; Mazelova et al., 2009a \\
\hline Rab8 & $\begin{array}{l}\text { C. elegans } \\
\text { Human, mouse }\end{array}$ & $\begin{array}{l}\text { Cell soma and dendrites } \\
\text { Cilia, Golgi }\end{array}$ & $\begin{array}{l}\text { Regulate ciliary } \\
\text { membrane sorting } \\
\text { or trafficking, } \\
\text { cilia formation }\end{array}$ & $\begin{array}{l}\text { Kaplan et al., 2010; } \\
\text { Nachury et al., } 2007\end{array}$ \\
\hline Rab11 & Human & Golgi and ciliary base & $\begin{array}{l}\text { Act upstream of } \\
\text { Rab8, cilia } \\
\text { formation }\end{array}$ & Knödler et al., 2010 \\
\hline Rab23 & Human, canine & Cilia & $\begin{array}{l}\text { Smo turnover in the } \\
\text { cilium }\end{array}$ & Yoshimura et al., 2007; Boehlke et al., 2010 \\
\hline Rab10 & $\begin{array}{l}\text { Mouse, rat, } \\
\text { Canine }\end{array}$ & Cilia base & $\begin{array}{l}\text { Redundant players } \\
\text { of Rab8? }\end{array}$ & Babbey et al., 2010 \\
\hline Rabl4 & $\begin{array}{l}\text { Chlamydomonas } \\
\text { Reinhardtii }\end{array}$ & Flagella & IFT & Qin et al., 2007 \\
\hline Rabl5 & C. elegans & Cilia & $\begin{array}{l}\text { Affect specific cilia } \\
\text { signaling } \\
\text { activities }\end{array}$ & Schafer et al., 2006 \\
\hline Ran & $\begin{array}{l}\text { Human, mouse and } \\
\text { canine }\end{array}$ & Cilia (GTP-bound Ran) & $\begin{array}{l}\text { Ciliary proteins } \\
\text { import }\end{array}$ & Dishinger et al., 2010 \\
\hline
\end{tabular}


GAPs, and effectors (such as adaptors, motors, kinases, and phosphatases) that bind specifically to the active form of small GTPases (Cherfils and Chardin, 1999; Bernards and Settleman, 2004; Winter-Vann and Casey, 2005; Stenmark, 2009). Biochemical identification and characterization of the binding partners of the cilia-related small GTPases would not only ensure the delineation of the poorly defined functional networks but also significantly enhance our understanding of the ciliary crosstalks among distinct small GTPases; for example, the regulation of the cilium entry of ciliary proteins by Rab11, Rab8, and Arf4 (Nachury et al., 2007; Mazelova et al., 2009a; Knödler et al., 2010), and the coordination of IFT integrity by ARL-3 and ARL-13 (Li et al., 2010). It's notable that multiple small GTPases could bind to different sites of the same effector, as proved by Rabin8 binding to both Rab11 and Rab8 in the regulation of rhodopsin cilary targeting (Knödler et al., 2010), and evidences from other non-cilia related events (Vitale et al., 1998; de Renzis et al., 2002; Sinka et al., 2008; Hayes et al., 2009).

Additionally, an important next step will be to generate transgenic animal models to study the in vivo functions of cilia-related small GTPases. Because of the essential role of cilia in early embryonic development and tissue pattern formation, it is prohibitively difficult to study the in vivo function of ciliogenic genes in live mammalian models (Nonaka et al., 1998; Essner et al., 2002). Accordingly, $\mathrm{Arl3}^{-/-}$and $\mathrm{Arl1}^{-/-}$ mice fail to thrive after birth (Schrick et al., 2006; Caspary et al., 2007). In this regard, the highly conserved ciliopathy genes, ciliogenesis pathway, and cilia sensory function of C. elegans make this organism a simple but powerful model for characterizing the physiologic roles of ciliary small GTPases in their native cellular environment (Barr, 2005). Many genes required for the formation, maintenance, and function of $C$. elegans sensory cilia have mammalian counterparts that, when mutated, cause ciliopathies (Barr, 2005) (Table 3). C. elegans is the only established multicellular eukaryotic model whose viability and normal development are not dependent on the existence of cilia. Thus, using of $C$. elegans enables the exploration of cilia biology in living animals. Recently, C. elegans has been successfully used to provide valuable insights into many ciliopathies, including ADPKD (Barr and Sternberg, 1999; Qin et al., 2001; Barr et al., 2001; Hu and Barr, 2005; Jauregui and Barr, 2005; Ou et al., 2005; Bae et al., 2006; Hu et al., 2006; Hu et al., 2007; Bae et al., 2008; Jauregui et al., 2008; Knobel et al., 2008), BBS (Ansley et al., 2003; Blacque et al., 2004; Blacque et al., 2005; Chen et al., 2006; Mak et al., 2006; Ou et al., 2007; Mukhopadhyay et al., 2008; Williams et al., 2010), Joubert Syndrome (Cevik et al., 2010; Li et al., 2010), MKS (Williams et al., 2008; Bialas et al., 2009; Williams et al., 2010), and NPHP (Jauregui and Barr, 2005; Wolf et al., 2005; Jauregui et al., 2008; Williams et al., 2008; Williams et al., 2010). C. elegans has many experimental advantages for analyzing the in vivo function of cilia genes: for example, various behavioral assays enable rapid testing of cilia sensory function; the existing battery of fluorescenceprotein-labeled ciliary markers; and the ability to perform timelapse microscopy of GFP-tagged IFT components or cargos, which make $C$. elegans a tractable model for the study of IFT machinery and cargo protein transport (Orozco et al., 1999; Signor et al., 1999a; Signor et al., 1999b; Snow et al., 2004; Qin et al., 2005); moreover, powerful genetics toolkits (e.g., genome-wide mutagenesis screens, transgenesis (Mello and Fire, 1995), and RNAi (Tabara et al., 1998)) are available to promptly identify and characterize genes involved in cilia formation and function.

Most ciliopathies share two common features: (1) cilialocalized gene products and (2) similar clinical manifestations, such as kidney cysts (reviewed in (Watnick and Germino, 2003). Determining the function and crosstalk of these ciliary proteins is pivotal to understanding disease pathology. Exploring the roles of small GTPases in ciliogenesis and ciliopathies has been advanced significantly by recent studies. The findings of these GTPases together with their regulators and effectors in ciliogenesis will broaden our understanding of how cilia develop and function in normal and

Table 3 The evolutionary conservation of ciliopathy loci

\begin{tabular}{llll}
\hline Disease & Loci & Worm homologs & Expression \\
\hline ADPKD & PKD1, PKD2 & lov-1, pkd-2 & Ciliated cells \\
ARPKD & PKHD & $n o$ & \\
Bardet-Biedle syndrome (BBS) & BBS1-12 & bbs-1,2,3,4,5,7,8,9,11 & Ciliated cells \\
Joubert syndrome & AHI1, NPHP1, CEP290, MKS3, & $n p h p-1, m k s-3$, arl-13, unc-26, & Ciliated cells \\
& RPGRIP1L, ARL13B, CC2D2A, & $k 07 g 5.3, c 09 g 5.8$ & \\
& InPP5E & & Ciliated cells \\
Meckel-Gruber syndrome (MKS) & MKS1, MKS3, CEP290, NPHP6, & $m k s-1, m k s-3$ & Ciliated cells \\
& CC2DA & $n p h p-1,2,4,7,8,9$ & Ciliated cells \\
Nephronophthisis & NPHP1-9 & ceofd-1 & Ciliated cells \\
Retinitis pigmentosa & OFD-1 & cerp2 & \\
\hline
\end{tabular}


pathological states and provide us new insights into the molecular basis of different ciliopathies. Most importantly, the fact that small GTPase itself and many of its effectors are enzymatically active molecules makes this group of proteins highly promising candidates for future therapeutic invention or phenotype reversion in ciliopathy treatment.

\section{ABBREVIATIONS}

ADPKD, autosomal dominant polycystic kidney disease; Arf, ADPribosylation factor; Arl proteins, Arf-like (Arl) proteins; BBS, Bardet-Biedl syndrome; CLS, ciliary localization signal; CNG, cyclic nucleotide-gated; GAPs, GTPase activating proteins; GEFs, guanine nucleotide exchange factors; IFT, intraflagellar transport; MKS, Meckel-Gruber syndrome; NLSs, nuclear localization signals; NPHP, nephronophthisis; PCP, Planar Cell Polarity; PDGFR, platelet-derived growth factor receptor; PKD, polycystic kidney disease; $\mathrm{PC2}$, polycystin-2; RFX, regulatory factor $\mathrm{X}$; TGN, trans-Golginetwork

\section{ACKNOWLEDGEMENTS}

We apologize to the scientists who made contributions to the field, but have not been cited due to space limitations. This research was funded by the National Institutes of Health grant 1R01DK090038 and the PKD Foundation Young Investigator Award 04YI09a to J. H.. J. H. is also supported by FULK Career Development Award, Zell PKD Research Fund, Upjohn PKD Research Fund, and Early Career Development Award from Mayo Clinic. J.H. is a member of NIH PKD translational Center at Mayo Clinic.

\section{REFERENCES}

Ansley, S.J., Badano, J.L., Blacque, O.E., Hill, J., Hoskins, B.E., Leitch, C.C., Kim, J.C., Ross, A.J., Eichers, E.R., Teslovich, T.M., et al. (2003). Basal body dysfunction is a likely cause of pleiotropic Bardet-Biedl syndrome. Nature 425, 628-633.

Avidor-Reiss, T., Maer, A.M., Koundakjian, E., Polyanovsky, A., Keil, T., Subramaniam, S., and Zuker, C.S. (2004). Decoding cilia function: defining specialized genes required for compartmentalized cilia biogenesis. Cell 117, 527-539.

Babbey, C.M., Bacallao, R.L., and Dunn, K.W. (2010). Rab10 associates with primary cilia and the exocyst complex in renal epithelial cells. Am J Physiol Renal Physiol 299, F495-F506.

Badano, J.L., Mitsuma, N., Beales, P.L., and Katsanis, N. (2006). The ciliopathies: an emerging class of human genetic disorders. Annu Rev Genomics Hum Genet 7, 125-148.

Bae, Y.K., Lyman-Gingerich, J., Barr, M.M., and Knobel, K.M. (2008). Identification of genes involved in the ciliary trafficking of C. elegans PKD-2. Dev Dyn 237, 2021-2029.

Bae, Y.K., Qin, H., Knobel, K.M., Hu, J., Rosenbaum, J.L., and Barr, M.M. (2006). General and cell-type specific mechanisms target TRPP2/PKD-2 to cilia. Development 133, 3859-3870.

Barr, M.M. (2005). Caenorhabditis elegans as a model to study renal development and disease: sexy cilia. J Am Soc Nephrol 16, 305-312.

Barr, M.M., DeModena, J., Braun, D., Nguyen, C.Q., Hall, D.H., and Sternberg, P.W. (2001). The Caenorhabditis elegans autosomal dominant polycystic kidney disease gene homologs lov-1 and pkd2 act in the same pathway. Curr Biol 11, 1341-1346.

Barr, M.M., and Sternberg, P.W. (1999). A polycystic kidney-disease gene homologue required for male mating behaviour in $\mathrm{C}$. elegans. Nature 401, 386-389.

Berbari, N.F., Lewis, J.S., Bishop, G.A., Askwith, C.C., and Mykytyn, K. (2008). Bardet-Biedl syndrome proteins are required for the localization of $\mathrm{G}$ protein-coupled receptors to primary cilia. Proc Natl Acad Sci U S A 105, 4242-4246.

Bernards, A., and Settleman, J. (2004). GAP control: regulating the regulators of small GTPases. Trends Cell Biol 14, 377-385.

Bialas, N.J., Inglis, P.N., Li, C., Robinson, J.F., Parker, J.D., Healey, M.P., Davis, E.E., Inglis, C.D., Toivonen, T., Cottell, D.C., et al. (2009). Functional interactions between the ciliopathy-associated Meckel syndrome 1 (MKS1) protein and two novel MKS1-related (MKSR) proteins. J Cell Sci 122, 611-624.

Blacque, O.E., Perens, E.A., Boroevich, K.A., Inglis, P.N., Li, C., Warner, A., Khattra, J., Holt, R.A., Ou, G., Mah, A.K., et al. (2005). Functional genomics of the cilium, a sensory organelle. Curr Biol 15, 935-941.

Blacque, O.E., Reardon, M.J., Li, C., McCarthy, J., Mahjoub, M.R., Ansley, S.J., Badano, J.L., Mah, A.K., Beales, P.L., Davidson, W. S., et al. (2004). Loss of C. elegans BBS-7 and BBS-8 protein function results in cilia defects and compromised intraflagellar transport. Genes Dev 18, 1630-1642.

Boehlke, C., Bashkurov, M., Buescher, A., Krick, T., John, A.K., Nitschke, R., Walz, G., and Kuehn, E.W. (2010). Differential role of Rab proteins in ciliary trafficking: Rab23 regulates smoothened levels. J Cell Sci 123, 1460-1467.

Boguski, M.S., and McCormick, F. (1993). Proteins regulating Ras and its relatives. Nature 366, 643-654.

Caspary, T., Larkins, C.E., and Anderson, K.V. (2007). The graded response to Sonic Hedgehog depends on cilia architecture. Dev Cell 12, 767-778.

Cevik, S., Hori, Y., Kaplan, O.I., Kida, K., Toivenon, T., Foley-Fisher, C., Cottell, D., Katada, T., Kontani, K., and Blacque, O.E. (2010). Joubert syndrome Arl13b functions at ciliary membranes and stabilizes protein transport in Caenorhabditis elegans. J Cell Biol 188, 953-969.

Chen, N., Mah, A., Blacque, O.E., Chu, J., Phgora, K., Bakhoum, M. W., Newbury, C.R., Khattra, J., Chan, S., Go, A., et al. (2006). Identification of ciliary and ciliopathy genes in Caenorhabditis elegans through comparative genomics. Genome Biol 7, R126.

Cherfils, J., and Chardin, P. (1999). GEFs: structural basis for their activation of small GTP-binding proteins. Trends Biochem Sci 24, 306-311.

Chiang, A.P., Nishimura, D., Searby, C., Elbedour, K., Carmi, R., Ferguson, A.L., Secrist, J., Braun, T., Casavant, T., Stone, E.M., et al. (2004). Comparative genomic analysis identifies an ADPribosylation factor-like gene as the cause of Bardet-Biedl syndrome (BBS3). Am J Hum Genet 75, 475-484.

Clarke, P.R., and Zhang, C. (2008). Spatial and temporal coordination of mitosis by Ran GTPase. Nat Rev Mol Cell Biol 9, 464-477.

Cuvillier, A., Redon, F., Antoine, J.C., Chardin, P., DeVos, T., and Merlin, G. (2000). LdARL-3A, a Leishmania promastigote-specific ADP-ribosylation factor-like protein, is essential for flagellum integrity. J Cell Sci 113, 2065-2074.

D'Souza-Schorey, C., and Chavrier, P. (2006). ARF proteins: roles in 
membrane traffic and beyond. Nat Rev Mol Cell Biol 7, 347-358.

de Renzis, S., Sönnichsen, B., and Zerial, M. (2002). Divalent Rab effectors regulate the sub-compartmental organization and sorting of early endosomes. Nat Cell Biol 4, 124-133.

Deretic, D., Huber, L.A., Ransom, N., Mancini, M., Simons, K., and Papermaster, D.S. (1995). rab8 in retinal photoreceptors may participate in rhodopsin transport and in rod outer segment disk morphogenesis. J Cell Sci 108, 215-224.

Deretic, D., Williams, A.H., Ransom, N., Morel, V., Hargrave, P.A., and Arendt, A. (2005). Rhodopsin C terminus, the site of mutations causing retinal disease, regulates trafficking by binding to ADPribosylation factor 4 (ARF4). Proc Natl Acad Sci U S A 102, 3301-3306.

Dishinger, J.F., Kee, H.L., Jenkins, P.M., Fan, S., Hurd, T.W., Hammond, J.W., Truong, Y.N., Margolis, B., Martens, J.R., and Verhey, K.J. (2010). Ciliary entry of the kinesin-2 motor KIF17 is regulated by importin-beta2 and RanGTP. Nat Cell Biol 12, 703-710.

Donovan, S., Shannon, K.M., and Bollag, G. (2002). GTPase activating proteins: critical regulators of intracellular signaling. Biochim Biophys Acta 1602, 23-45.

Duldulao, N.A., Lee, S., and Sun, Z. (2009). Cilia localization is essential for in vivo functions of the Joubert syndrome protein Arl13b/Scorpion. Development 136, 4033-4042.

Eggenschwiler, J.T., Bulgakov, O.V., Qin, J., Li, T., and Anderson, K.V. (2006). Mouse Rab23 regulates hedgehog signaling from smoothened to Gli proteins. Dev Biol 290, 1-12.

Eley, L., Yates, L.M., and Goodship, J.A. (2005). Cilia and disease. Curr Opin Genet Dev 15, 308-314.

Essner, J.J., Vogan, K.J., Wagner, M.K., Tabin, C.J., Yost, H.J., and Brueckner, M. (2002). Conserved function for embryonic nodal cilia. Nature 418, 37-38.

Evans, R.J., Schwarz, N., Nagel-Wolfrum, K., Wolfrum, U., Hardcastle, A.J., and Cheetham, M.E. (2010). The retinitis pigmentosa protein RP2 links pericentriolar vesicle transport between the Golgi and the primary cilium. Hum Mol Genet 19, 1358-1367.

Fan, Y., Esmail, M.A., Ansley, S.J., Blacque, O.E., Boroevich, K., Ross, A.J., Moore, S.J., Badano, J.L., May-Simera, H., Compton, D.S., et al. (2004). Mutations in a member of the Ras superfamily of small GTP-binding proteins causes Bardet-Biedl syndrome. Nat Genet 36, 989-993.

Fielding, A.B., Schonteich, E., Matheson, J., Wilson, G., Yu, X., Hickson, G.R., Srivastava, S., Baldwin, S.A., Prekeris, R., and Gould, G.W. (2005). Rab11-FIP3 and FIP4 interact with Arf6 and the exocyst to control membrane traffic in cytokinesis. EMBO J 24 , 3389-3399.

Fliegauf, M., Benzing, T., and Omran, H. (2007). When cilia go bad: cilia defects and ciliopathies. Nat Rev Mol Cell Biol 8, 880-893.

Follit, J.A., Li, L., Vucica, Y., and Pazour, G.J. (2010). The cytoplasmic tail of fibrocystin contains a ciliary targeting sequence. J Cell Biol 188, 21-28.

Fukushige, T., Siddiqui, Z.K., Chou, M., Culotti, J.G., Gogonea, C.B., Siddiqui, S.S., and Hamelin, M. (1999). MEC-12, an alpha-tubulin required for touch sensitivity in C. elegans. J Cell Sci 112, 395- 403.

Geng, L., Okuhara, D., Yu, Z., Tian, X., Cai, Y., Shibazaki, S., and Somlo, S. (2006). Polycystin-2 traffics to cilia independently of polycystin-1 by using an N-terminal RVxP motif. J Cell Sci 119, 1383-1395.

Gerdes, J.M., Davis, E.E., and Katsanis, N. (2009). The vertebrate primary cilium in development, homeostasis, and disease. Cell 137, 32-45.

Gherman, A., Davis, E.E., and Katsanis, N. (2006). The ciliary proteome database: an integrated community resource for the genetic and functional dissection of cilia. Nat Genet 38, 961-962.

Goetz, S.C., and Anderson, K.V. (2010). The primary cilium: a signalling centre during vertebrate development. Nat Rev Genet 11, 331-344.

Grayson, C., Bartolini, F., Chapple, J.P., Willison, K.R., Bhamidipati, A., Lewis, S.A., Luthert, P.J., Hardcastle, A.J., Cowan, N.J., and Cheetham, M.E. (2002). Localization in the human retina of the $X-$ linked retinitis pigmentosa protein RP2, its homologue cofactor $\mathrm{C}$ and the RP2 interacting protein Arl3. Hum Mol Genet 11, 3065-3074

Grozinger, C.M., Hassig, C.A., and Schreiber, S.L. (1999). Three proteins define a class of human histone deacetylases related to yeast Hda1p. Proc Natl Acad Sci U S A 96, 4868-4873.

Guo, W., Roth, D., Walch-Solimena, C., and Novick, P. (1999). The exocyst is an effector for Sec $4 p$, targeting secretory vesicles to sites of exocytosis. EMBO J 18, 1071-1080.

Hayes, G.L., Brown, F.C., Haas, A.K., Nottingham, R.M., Barr, F.A., and Pfeffer, S.R. (2009). Multiple Rab GTPase binding sites in GCC185 suggest a model for vesicle tethering at the trans-Golgi. Mol Biol Cell 20, 209-217.

He, B., and Guo, W. (2009). The exocyst complex in polarized exocytosis. Curr Opin Cell Biol 21, 537-542.

Hori, Y., Kobayashi, T., Kikko, Y., Kontani, K., and Katada, T. (2008). Domain architecture of the atypical Arf-family GTPase Arl13b involved in cilia formation. Biochem Biophys Res Commun 373, 119-124.

Hu, J., Bae, Y.K., Knobel, K.M., and Barr, M.M. (2006). Casein kinase II and calcineurin modulate TRPP function and ciliary localization. Mol Biol Cell 17, 2200-2211.

$\mathrm{Hu}$, J., and Barr, M.M. (2005). ATP-2 interacts with the PLAT domain of LOV-1 and is involved in Caenorhabditis elegans polycystin signaling. Mol Biol Cell 16, 458-469.

Hu, J., Wittekind, S.G., and Barr, M.M. (2007). STAM and Hrs downregulate ciliary TRP receptors. Mol Biol Cell 18, 3277-3289.

Huangfu, D., and Anderson, K.V. (2005). Cilia and Hedgehog responsiveness in the mouse. Proc Natl Acad Sci U S A 102, $11325-11330$

Hubbert, C., Guardiola, A., Shao, R., Kawaguchi, Y., Ito, A., Nixon, A., Yoshida, M., Wang, X.F., and Yao, T.P. (2002). HDAC6 is a microtubule-associated deacetylase. Nature 417, 455-458.

Jaffe, A.B., and Hall, A. (2005). Rho GTPases: biochemistry and biology. Annu Rev Cell Dev Biol 21, 247-269.

Jauregui, A.R., and Barr, M.M. (2005). Functional characterization of the C. elegans nephrocystins NPHP-1 and NPHP-4 and their role in cilia and male sensory behaviors. Exp Cell Res 305, 333-342.

Jauregui, A.R., Nguyen, K.C., Hall, D.H., and Barr, M.M. (2008). The Caenorhabditis elegans nephrocystins act as global modifiers of cilium structure. J Cell Biol 180, 973-988.

Jenkins, D., Seelow, D., Jehee, F.S., Perlyn, C.A., Alonso, L.G., Bueno, D.F., Donnai, D., Josifova, D., Mathijssen, I.M., Morton, J. 
E., et al. (2007). RAB23 mutations in Carpenter syndrome imply an unexpected role for hedgehog signaling in cranial-suture development and obesity. Am J Hum Genet 80, 1162-1170.

Jenkins, P.M., Hurd, T.W., Zhang, L., McEwen, D.P., Brown, R.L., Margolis, B., Verhey, K.J., and Martens, J.R. (2006). Ciliary targeting of olfactory CNG channels requires the CNGB1b subunit and the kinesin-2 motor protein, KIF17. Curr Biol 16, 1211-1216.

Jin, H., White, S.R., Shida, T., Schulz, S., Aguiar, M., Gygi, S.P., Bazan, J.F., and Nachury, M.V. (2010). The conserved BardetBiedl syndrome proteins assemble a coat that traffics membrane proteins to cilia. Cell 141, 1208-1219.

Kaplan, O.I., Molla-Herman, A., Cevik, S., Ghossoub, R., Kida, K., Kimura, Y., Jenkins, P., Martens, J.R., Setou, M., Benmerah, A.,et al. (2010). The AP-1 clathrin adaptor facilitates cilium formation and functions with RAB-8 in C. elegans ciliary membrane transport. J Cell Sci 123, 3966-3977.

Kim, J., Krishnaswami, S.R., and Gleeson, J.G. (2008). CEP290 interacts with the centriolar satellite component PCM-1 and is required for Rab8 localization to the primary cilium. Hum Mol Genet 17, 3796-3805.

Knobel, K.M., Peden, E.M., and Barr, M.M. (2008). Distinct protein domains regulate ciliary targeting and function of $C$. elegans PKD2. Exp Cell Res 314, 825-833.

Knödler, A., Feng, S., Zhang, J., Zhang, X., Das, A., Peränen, J., and Guo, W. (2010). Coordination of Rab8 and Rab11 in primary ciliogenesis. Proc Natl Acad Sci U S A 107, 6346-6351.

Kovacs, J.J., Murphy, P.J., Gaillard, S., Zhao, X., Wu, J.T., Nicchitta, C.V., Yoshida, M., Toft, D.O., Pratt, W.B., and Yao, T.P. (2005). HDAC6 regulates Hsp90 acetylation and chaperone-dependent activation of glucocorticoid receptor. Mol Cell 18, 601-607.

Kozminski, K.G., Forscher, P., and Rosenbaum, J.L. (1998). Three flagellar motilities in Chlamydomonas unrelated to flagellar beating. Video supplement. Cell Motil Cytoskeleton 39, 347-348.

Lechtreck, K.F., Johnson, E.C., Sakai, T., Cochran, D., Ballif, B.A., Rush, J., Pazour, G.J., Ikebe, M., and Witman, G.B. (2009). The Chlamydomonas reinhardtii BBSome is an IFT cargo required for export of specific signaling proteins from flagella. J Cell Biol 187, 1117-1132.

Li, Y., Wei, Q., Zhang, Y., Ling, K., and Hu, J. (2010). The small GTPases ARL-13 and ARL-3 coordinate intraflagellar transport and ciliogenesis. J Cell Biol 189, 1039-1051.

Liu, Q., Tan, G., Levenkova, N., Li, T., Pugh, E.N. Jr, Rux, J.J., Speicher, D.W., and Pierce, E.A. (2007). The proteome of the mouse photoreceptor sensory cilium complex. Mol Cell Proteomics 6, 1299-1317.

Loktev, A.V., Zhang, Q., Beck, J.S., Searby, C.C., Scheetz, T.E., Bazan, J.F., Slusarski, D.C., Sheffield, V.C., Jackson, P.K., and Nachury, M.V. (2008). A BBSome subunit links ciliogenesis, microtubule stability, and acetylation. Dev Cell 15, 854-865.

Lowy, D.R., and Willumsen, B.M. (1993). Function and regulation of ras. Annu Rev Biochem 62, 851-891.

Lundquist, E.A. (2006). Small GTPases. WormBook Jan 17, 1-18. http://www.wormbook.org.

Mak, H.Y., Nelson, L.S., Basson, M., Johnson, C.D., and Ruvkun, G. (2006). Polygenic control of Caenorhabditis elegans fat storage. Nat Genet 38, 363-368.

Marshall, W.F. (2008). The cell biological basis of ciliary disease. J Cell Biol 180, 17-21.
May, S.R., Ashique, A.M., Karlen, M., Wang, B., Shen, Y., Zarbalis, K., Reiter, J., Ericson, J., and Peterson, A.S. (2005). Loss of the retrograde motor for IFT disrupts localization of Smo to cilia and prevents the expression of both activator and repressor functions of Gli. Dev Biol 287, 378-389.

Mazelova, J., Astuto-Gribble, L., Inoue, H., Tam, B.M., Schonteich, E., Prekeris, R., Moritz, O.L., Randazzo, P.A., and Deretic, D. (2009a). Ciliary targeting motif $\mathrm{VxPx}$ directs assembly of a trafficking module through Arf4. EMBO J 28, 183-192.

Mazelova, J., Ransom, N., Astuto-Gribble, L., Wilson, M.C., and Deretic, D. (2009b). Syntaxin 3 and SNAP-25 pairing, regulated by omega-3 docosahexaenoic acid, controls the delivery of rhodopsin for the biogenesis of cilia-derived sensory organelles, the rod outer segments. J Cell Sci 122, 2003-2013.

Mello, C., and Fire, A. (1995). DNA transformation. Methods Cell Biol 48, 451-482.

Moritz, O.L., Tam, B.M., Hurd, L.L., Peränen, J., Deretic, D., and Papermaster, D.S. (2001). Mutant rab8 Impairs docking and fusion of rhodopsin-bearing post-Golgi membranes and causes cell death of transgenic Xenopus rods. Mol Biol Cell 12, 2341-2351.

Mukhopadhyay, S., Lu, Y., Shaham, S., and Sengupta, P. (2008). Sensory signaling-dependent remodeling of olfactory cilia architecture in C. elegans. Dev Cell 14, 762-774.

Myers, K.R., and Casanova, J.E. (2008). Regulation of actin cytoskeleton dynamics by Arf-family GTPases. Trends Cell Biol 18, 184-192.

Nachury, M.V., Loktev, A.V., Zhang, Q., Westlake, C.J., Peränen, J., Merdes, A., Slusarski, D.C., Scheller, R.H., Bazan, J.F., Sheffield, V.C., et al. (2007). A core complex of BBS proteins cooperates with the GTPase Rab8 to promote ciliary membrane biogenesis. Cell 129, 1201-1213.

Nonaka, S., Tanaka, Y., Okada, Y., Takeda, S., Harada, A., Kanai, Y., Kido, M., and Hirokawa, N. (1998). Randomization of left-right asymmetry due to loss of nodal cilia generating leftward flow of extraembryonic fluid in mice lacking KIF3B motor protein. Cell 95, 829-837.

Omori, Y., Zhao, C., Saras, A., Mukhopadhyay, S., Kim, W., Furukawa, T., Sengupta, P., Veraksa, A., and Malicki, J. (2008). Elipsa is an early determinant of ciliogenesis that links the IFT particle to membrane-associated small GTPase Rab8. Nat Cell Biol 10, 437-444.

Orozco, J.T., Wedaman, K.P., Signor, D., Brown, H., Rose, L., and Scholey, J.M. (1999). Movement of motor and cargo along cilia. Nature 398, 674

Ou, G., Blacque, O.E., Snow, J.J., Leroux, M.R., and Scholey, J.M. (2005). Functional coordination of intraflagellar transport motors. Nature 436, 583-587.

Ou, G., Koga, M., Blacque, O.E., Murayama, T., Ohshima, Y., Schafer, J.C., Li, C., Yoder, B.K., Leroux, M.R., and Scholey, J.M. (2007). Sensory ciliogenesis in Caenorhabditis elegans: assignment of IFT components into distinct modules based on transport and phenotypic profiles. Mol Biol Cell 18, 1554-1569.

Oztan, A., Silvis, M., Weisz, O.A., Bradbury, N.A., Hsu, S.C., Goldenring, J.R., Yeaman, C., and Apodaca, G. (2007). Exocyst requirement for endocytic traffic directed toward the apical and basolateral poles of polarized MDCK cells. Mol Biol Cell 18, 3978-3992.

Peden, E.M., and Barr, M.M. (2005). The KLP-6 kinesin is required for 
male mating behaviors and polycystin localization in Caenorhabditis elegans. Curr Biol 15, 394-404.

Pedersen, L.B., and Rosenbaum, J.L. (2008). Intraflagellar transport (IFT) role in ciliary assembly, resorption and signalling. Curr Top Dev Biol 85, 23-61.

Pereira-Leal, J.B., and Seabra, M.C. (2001). Evolution of the Rab family of small GTP-binding proteins. J Mol Biol 313, 889-901.

Prigent, M., Dubois, T., Raposo, G., Derrien, V., Tenza, D., Rossé, C., Camonis, J., and Chavrier, P. (2003). ARF6 controls postendocytic recycling through its downstream exocyst complex effector. J Cell Biol 163, 1111-1121.

Pugacheva, E.N., Jablonski, S.A., Hartman, T.R., Henske, E.P., and Golemis, E.A. (2007). HEF1-dependent Aurora A activation induces disassembly of the primary cilium. Cell 129, 1351-1363.

Qin, H., Burnette, D.T., Bae, Y.K., Forscher, P., Barr, M.M., and Rosenbaum, J.L. (2005). Intraflagellar transport is required for the vectorial movement of TRPV channels in the ciliary membrane. Curr Biol 15, 1695-1699.

Qin, H., Rosenbaum, J.L., and Barr, M.M. (2001). An autosomal recessive polycystic kidney disease gene homolog is involved in intraflagellar transport in $\mathrm{C}$. elegans ciliated sensory neurons. Curr Biol 11, 457-461

Qin, H., Wang, Z., Diener, D., and Rosenbaum, J. (2007). Intraflagellar transport protein 27 is a small $\mathrm{G}$ protein involved in cell-cycle control. Curr Biol 17, 193-202.

Reed, N.A., Cai, D., Blasius, T.L., Jih, G.T., Meyhofer, E., Gaertig, J., and Verhey, K.J. (2006). Microtubule acetylation promotes kinesin1 binding and transport. Curr Biol 16, 2166-2172.

Reuther, G.W., and Der, C.J. (2000). The Ras branch of small GTPases: Ras family members don't fall far from the tree. Curr Opin Cell Biol 12, 157-165.

Rogers, K.K., Wilson, P.D., Snyder, R.W., Zhang, X., Guo, W., Burrow, C.R., and Lipschutz, J.H. (2004). The exocyst localizes to the primary cilium in MDCK cells. Biochem Biophys Res Commun 319, 138-143.

Rosenbaum, J.L., and Witman, G.B. (2002). Intraflagellar transport. Nat Rev Mol Cell Biol 3, 813-825.

Schafer, J.C., Winkelbauer, M.E., Williams, C.L., Haycraft, C.J., Desmond, R.A., and Yoder, B.K. (2006). IFTA-2 is a conserved cilia protein involved in pathways regulating longevity and dauer formation in Caenorhabditis elegans. J Cell Sci 119, 4088-4100.

Scholey, J.M. (2008). Intraflagellar transport motors in cilia: moving along the cell's antenna. J Cell Biol 180, 23-29.

Scholey, J.M., and Anderson, K.V. (2006). Intraflagellar transport and cilium-based signaling. Cell 125, 439-442.

Schrick, J.J., Vogel, P., Abuin, A., Hampton, B., and Rice, D.S. (2006). ADP-ribosylation factor-like 3 is involved in kidney and photoreceptor development. Am J Pathol 168, 1288-1298.

Signor, D., Wedaman, K.P., Orozco, J.T., Dwyer, N.D., Bargmann, C. I., Rose, L.S., and Scholey, J.M. (1999a). Role of a class DHC1b dynein in retrograde transport of IFT motors and IFT raft particles along cilia, but not dendrites, in chemosensory neurons of living Caenorhabditis elegans. J Cell Biol 147, 519-530.

Signor, D., Wedaman, K.P., Rose, L.S., and Scholey, J.M. (1999b). Two heteromeric kinesin complexes in chemosensory neurons and sensory cilia of Caenorhabditis elegans. Mol Biol Cell 10, 345-360.

Singla, V., and Reiter, J.F. (2006). The primary cilium as the cell's antenna: signaling at a sensory organelle. Science 313, 629-633.
Sinka, R., Gillingham, A.K., Kondylis, V., and Munro, S. (2008). Golgi coiled-coil proteins contain multiple binding sites for Rab family $G$ proteins. J Cell Biol 183, 607-615.

Snow, J.J., Ou, G., Gunnarson, A.L., Walker, M.R., Zhou, H.M., BrustMascher, I., and Scholey, J.M. (2004). Two anterograde intraflagellar transport motors cooperate to build sensory cilia on C. elegans neurons. Nat Cell Biol 6, 1109-1113.

Stenmark, H. (2009). Rab GTPases as coordinators of vesicle traffic. Nat Rev Mol Cell Biol 10, 513-525.

Stenmark, H., Vitale, G., Ullrich, O., and Zerial, M. (1995). Rabaptin-5 is a direct effector of the small GTPase Rab5 in endocytic membrane fusion. Cell 83, 423-432.

Stewart, M. (2007). Molecular mechanism of the nuclear protein import cycle. Nat Rev Mol Cell Biol 8, 195-208.

Swoboda, P., Adler, H.T., and Thomas, J.H. (2000). The RFX-type transcription factor DAF-19 regulates sensory neuron cilium formation in C. elegans. Mol Cell 5, 411-421.

Tabara, H., Grishok, A., and Mello, C.C. (1998). RNAi in C. elegans: soaking in the genome sequence. Science 282, 430-431.

Takaki, E., Fujimoto, M., Nakahari, T., Yonemura, S., Miyata, Y., Hayashida, N., Yamamoto, K., Vallee, R.B., Mikuriya, T., Sugahara, K., et al. (2007). Heat shock transcription factor 1 is required for maintenance of ciliary beating in mice. J Biol Chem 282, 37285-37292.

Tran, P.V., Haycraft, C.J., Besschetnova, T.Y., Turbe-Doan, A., Stottmann, R.W., Herron, B.J., Chesebro, A.L., Qiu, H., Scherz, P.J., Shah, J.V., et al. (2008). THM1 negatively modulates mouse sonic hedgehog signal transduction and affects retrograde intraflagellar transport in cilia. Nat Genet 40, 403-410.

Tsang, W.Y., Bossard, C., Khanna, H., Peränen, J., Swaroop, A., Malhotra, V., and Dynlacht, B.D. (2008). CP110 suppresses primary cilia formation through its interaction with CEP290, a protein deficient in human ciliary disease. Dev Cell 15, 187-197.

Vitale, G., Rybin, V., Christoforidis, S., Thornqvist, P., McCaffrey, M., Stenmark, H., and Zerial, M. (1998). Distinct Rab-binding domains mediate the interaction of Rabaptin-5 with GTP-bound Rab4 and Rab5. EMBO J 17, 1941-1951.

Watnick, T., and Germino, G. (2003). From cilia to cyst. Nat Genet 34, 355-356.

Weis, K. (2003). Regulating access to the genome: nucleocytoplasmic transport throughout the cell cycle. Cell 112, 441-451.

Wennerberg, K., Rossman, K.L., and Der, C.J. (2005). The Ras superfamily at a glance. J Cell Sci 118, 843-846.

Wiens, C.J., Tong, Y., Esmail, M.A., Oh, E., Gerdes, J.M., Wang, J., Tempel, W., Rattner, J.B., Katsanis, N., Park, H.W., et al. (2010). Bardet-Biedl syndrome-associated small GTPase ARL6 (BBS3) functions at or near the ciliary gate and modulates Wnt signaling. J Biol Chem 285, 16218-16230.

Williams, C.L., Masyukova, S.V., and Yoder, B.K. (2010). Normal ciliogenesis requires synergy between the cystic kidney disease genes MKS-3 and NPHP-4. J Am Soc Nephrol 21, 782-793.

Williams, C.L., Winkelbauer, M.E., Schafer, J.C., Michaud, E.J., and Yoder, B.K. (2008). Functional redundancy of the B9 proteins and nephrocystins in Caenorhabditis elegans ciliogenesis. Mol Biol Cell 19, 2154-2168.

Winter-Vann, A.M., and Casey, P.J. (2005). Post-prenylationprocessing enzymes as new targets in oncogenesis. Nat Rev Cancer 5, 405-412. 
Wolf, M.T., Lee, J., Panther, F., Otto, E.A., Guan, K.L., and Hildebrandt, F. (2005). Expression and phenotype analysis of the nephrocystin-1 and nephrocystin-4 homologs in Caenorhabditis elegans. J Am Soc Nephrol 16, 676-687.

Wu, S., Mehta, S.Q., Pichaud, F., Bellen, H.J., and Quiocho, F.A. (2005). Sec15 interacts with Rab11 via a novel domain and affects Rab11 localization in vivo. Nat Struct Mol Biol 12, 879-885.

Yoshimura, S., Egerer, J., Fuchs, E., Haas, A.K., and Barr, F.A. (2007). Functional dissection of Rab GTPases involved in primary cilium formation. J Cell Biol 178, 363-369.

Zaghloul, N.A., and Katsanis, N. (2009). Mechanistic insights into Bardet-Biedl syndrome, a model ciliopathy. J Clin Invest 119 , 428-437.

Zerial, M., and McBride, H. (2001). Rab proteins as membrane organizers. Nat Rev Mol Cell Biol 2, 107-117.
Zhang, X., Yuan, Z., Zhang, Y., Yong, S., Salas-Burgos, A., Koomen, J., Olashaw, N., Parsons, J.T., Yang, X.J., Dent, S.R., et al. (2007). HDAC6 modulates cell motility by altering the acetylation level of cortactin. Mol Cell 27, 197-213.

Zhang, X.M., Ellis, S., Sriratana, A., Mitchell, C.A., and Rowe, T. (2004). Sec15 is an effector for the Rab11 GTPase in mammalian cells. J Biol Chem 279, 43027-43034.

Zheng, Y. (2004). G protein control of microtubule assembly. Annu Rev Cell Dev Biol 20, 867-894.

Zuo, X., Guo, W., and Lipschutz, J.H. (2009). The exocyst protein Sec10 is necessary for primary ciliogenesis and cystogenesis in vitro. Mol Biol Cell 20, 2522-2529.

Zhou, C., Cunningham, L., Marcus, A.I., Li, Y., and Kahn, R.A. (2006). Arl2 and Arl3 regulate different microtubule-dependent processes. Mol Biol Cell 17, 2476-2487. 